See discussions, stats, and author profiles for this publication at: https://www.researchgate.net/publication/325306252

\title{
Integrated $\mathrm{SiN}$ on SOI dual photonic devices for advanced datacom solutions
}

Conference Paper · May 2018

DOI: $10.1117 / 12.2306160$

CITATIONS

5

18 authors, including:

Sylvain Guerber

Université Paris-Saclay

31 PUBLICATIONS 106 CITATIONS

SEE PROFILE

Diego Perez-Galacho

Universitat Politècnica de València

92 PUBLICATIONS 611 CITATIONS

SEE PROFILE
READS

311

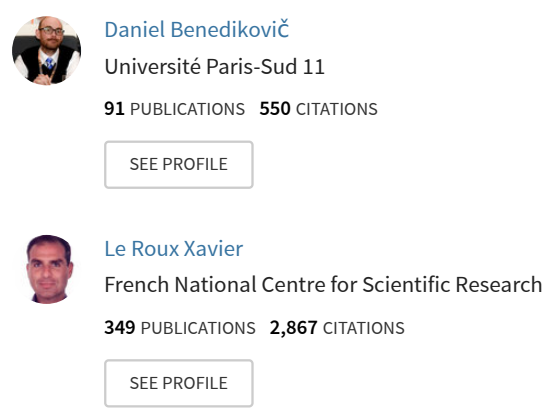

Some of the authors of this publication are also working on these related projects:

NTU-ST-Microelectronics Project View project

Slovak Grant Agency VEGA 1/1271 View project 


\title{
Integrated SiN on SOI dual photonic devices for advanced datacom solutions
}

\author{
Sylvain Guerber ${ }^{\mathrm{a}}$,, Carlos Alonso-Ramos ${ }^{\mathrm{b}}$, Daniel Benedikovic ${ }^{\mathrm{b}}$, Diego Perez-Galacho $^{\mathrm{b}}$, Xavier Le \\ Roux $^{\mathrm{b}}$, Nathalie Vulliet ${ }^{\mathrm{a}}$, Sébastien Crémer ${ }^{\mathrm{a}}$, Laurène Babaud ${ }^{\mathrm{a}}$, Jonathan Planchot ${ }^{\mathrm{a}}$, Daniel Benoit ${ }^{\mathrm{a}}$, \\ Paul Chantraine ${ }^{\mathrm{a}}$, François Leverd ${ }^{\mathrm{a}}$, Delia Ristoiu ${ }^{\mathrm{a}}$, Philippe Grosse ${ }^{\mathrm{c}}$, Delphine Marris-Morini ${ }^{\mathrm{b}}$, \\ Laurent Vivien $^{\mathrm{b}}$, Charles Baudot ${ }^{\mathrm{a}}$, Frédéric Boeuf $^{\mathrm{a}}$ \\ ${ }^{\mathrm{a}}$ TR\&D - STMicroelectronics SAS - 850 rue Jean Monnet - 38920 CROLLES France ; \\ ${ }^{\mathrm{b}}$ Centre for Nanoscience and Nanotechnology - Université Paris Saclay - CNRS - Université Paris \\ Sud - 91405 ORSAY FRANCE; \\ ${ }^{\mathrm{c} C E A}$, LETI, MINATEC Campus, CEA-Grenoble, F-38054 Grenoble, France
}

\begin{abstract}
We report on the co-integration of an additional passive layer within a Silicon Photonic chip for advanced passive devices. Being a CMOS compatible material, Silicon Nitride (SiN) appears as an attractive candidate. With a moderate refractive index contrast compared to SOI, SiN based devices would be intrinsically much more tolerant to fabrication errors while keeping a reasonable footprint. In addition, it's seven times lower thermo-optical coefficient, relatively to Silicon, could lead to thermal-tuning free components. The co-integration of SiN on SOI has been explored in ST $300 \mathrm{~mm} R \& D$ photonic platform DAPHNE and is presented in this paper. Surface roughness of the SiN films have been characterized through Atomic Force Microscopy (AFM) showing an RMS roughness below $2 \mathrm{~nm}$. The film thickness uniformity have been evaluated by ellipsometry revealing a three-sigma of $21 \mathrm{~nm}$. Statistical measurements have been performed on basic key building blocks such as SiN strip waveguide showing propagation loss below $0.7 \mathrm{~dB} / \mathrm{cm}$ and $40 \mu \mathrm{m}$ radius bends with losses below $0.02 \mathrm{~dB} / 90^{\circ}$. A compact Si-SiN transition taper was developed and statistically measured showing insertion losses below $0.17 \mathrm{~dB} /$ transition on the whole O-band wavelength range. Moreover, advanced WDM devices such as wavelength-stabilized directional couplers (WSDC) have been developed.
\end{abstract}

Keywords: Silicon photonics, Silicon Nitride, Coarse Wavelength Division Multiplexing, co-integration, Datacom.

\section{INTRODUCTION}

During the last decades, Silicon Photonics has demonstrated capability to provide most elementary functions for optical data communication: modulation ${ }^{1}$, detection ${ }^{2}$ and chip-fiber coupling ${ }^{3,4}$. Moreover, as a CMOS compatible technology, Silicon Photonics pledges for low cost and high volume production. Those promising perspectives together with an increasing demand of bandwidth, driven by Cloud applications ${ }^{5}$, have led to a strong expansion of the field over the last ten years. This paved the way to the emergence of industrial platforms ${ }^{6,7}$ creating the first generation of integrated optical transceivers in a Parallel Single Mode (PSM) scheme. Perspectives in bandwidth requirements and cable length are reaching such high densities and long distances that PSM will soon be no more relevant for some applications since its cost is raising up due to optical fiber multiplication. A promising solution already widely used in discrete photonics and which consists of using several optical data channels at different wavelengths is called Wavelength Division Multiplexing (WDM). Therefore, by increasing the spectral density, the bandwidth of a single optical fiber is raised to a much higher level. Such solutions have already been implemented in Silicon, demonstrating advanced devices such as multiplexer/demultiplexer (MUX/DEMUX) ${ }^{8-10}$, polarization splitters ${ }^{11}$ and rotators ${ }^{12}$. However, the massive production of a platform with such advanced devices makes the fabrication extremely challenging. An alternative solution based on 3D assembly of glass platform to realize the sensitive WDM functions has been proposed $^{13}$. But the prohibitive packaging and assembly costs makes it not relevant for large volume industrialization.

Consequently, our proposed approach consists of co-integrating within the Silicon platform a different photonic layer to provide low-cost and process tolerant advanced passive devices such as MUX/DEMUX. Being a CMOS compatible and well known material, Silicon Nitride ( $\mathrm{SiN}$ ) appears as an attractive candidate. With a moderate refractive index contrast compared to SOI, SiN based devices would be intrinsically much more tolerant to fabrication errors while keeping a reasonable footprint. In addition, it's seven times lower thermo-optical coefficient ${ }^{14-15}$, relatively to Silicon, 
could lead to thermal-tuning free MUX/DEMUX greatly reducing the system power consumption. Furthermore, Silicon Nitride could offer new functionalities for advanced applications. Among which can be listed bio sensing ${ }^{16}$ and efficient non-linear optical functions ${ }^{17,18}$. This co-integration of SiN on SOI has been explored in ST 300mm R\&D photonic platform DAPHNE ${ }^{19}$ and will be presented in this paper. Particular attention have been paid on devices characterization for O-band Coarse WDM (CWDM) applications.

\section{CO-INTEGRATION OF THE SILICON NITRIDE PHOTONICS LAYER}

The ST platform DAPHNE ${ }^{19}$ is a $300 \mathrm{~mm}$ SOI-based R\&D tool. This technological environment is particularly suited for WDM systems targeting datacom applications thanks to silicon passive devices with multilevel patterning, Germanium based photodetectors, carrier depletion modulators and a 4 level interconnection back end. Figure 1(a) depict a cross section schematic view of a DAPHNE typical photonic chip. As shown, the SiN platform have been integrated within the contact layer allowing an efficient optical power coupling with the SOI-based photonic devices. Figure 1(b) shows a Cross-Section Transmission Electron Microscope (XTEM) picture of a Si-SiN adiabatic transition taper revealing a good alignment between both layers and flat SiN bottom surface thanks to a Chemical-Mechanical Polishing (CMP) step after the SOI-based devices encapsulation by silicon dioxide.

(a)

(b)

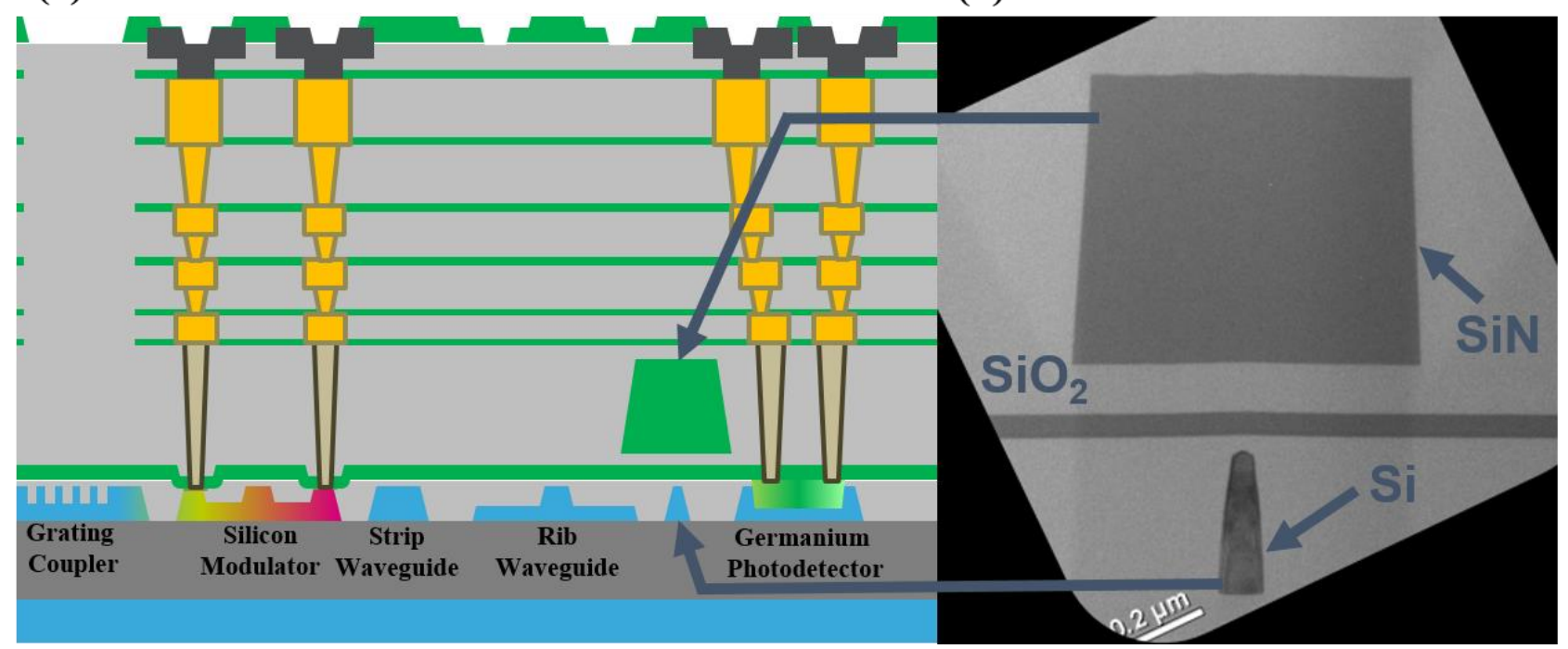

Figure 1. SOI-based photonic chip with co-integration of Silicon Nitride within the contact level. (a) Schematic cut view of the photonic chip. (b) XTEM view of a Si-SiN transition taper.

The SiN process starts by the deposition of a 610nm thick SiN film in prevision of a nitride-stop CMP step giving a final SiN film thickness of 600nm. The Silicon Nitride film have been deposited by low temperature Plasma Enhanced Chemical Vapor Deposition (PECVD) with a standard ammoniac-based chemistry. The as deposited film thickness have been investigated by surface ellipsometry as shown on Figure 2(a). A mean value of 608nm have been obtained with a three-sigma of $21.6 \mathrm{~nm}$. Such low standard-deviation suggests stable and uniform photonic devices. As surface roughness appears to be one of the main cause of waveguides propagation losses, SiN film top surface have been characterized through Atomic Force Microscope (AFM) measurements as shown on Figure 2(b). A smooth interface is obtained with an arithmetic average deviation of the surface height, $\mathrm{R}_{\mathrm{a}}$, of $1.53 \mathrm{~nm}$ indicating that the $\mathrm{SiN}$ film does not induce additional roughness. Indeed, those measurements were performed on a SiN film deposited on a $\mathrm{Si}$ crystalline wafer that present a remarkably low surface roughness. However, the roughness of the substrate on which the SiN film have been deposited seems to play a large role on the overall propagation losses ${ }^{20}$. To complete the SiN material characterization, the thermal dependence have been measured through the temperature drift of a Bragg filter resonance. A value of $20 \mathrm{pm} /{ }^{\circ} \mathrm{C}$ have been measured giving a thermo-optical coefficient of $4.3 * 10^{-5} \mathrm{~K}^{-1}$ which is in good agreement with previously reported values ${ }^{14,15}$. 
(a)

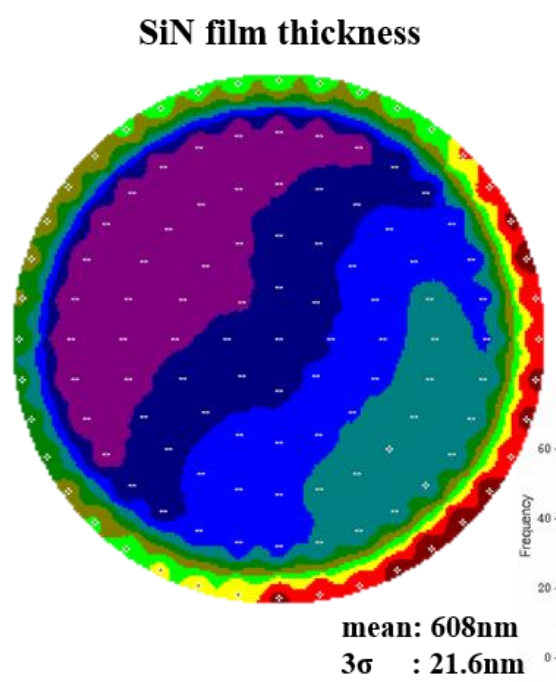

(b)

\section{SiN film top surface roughness}

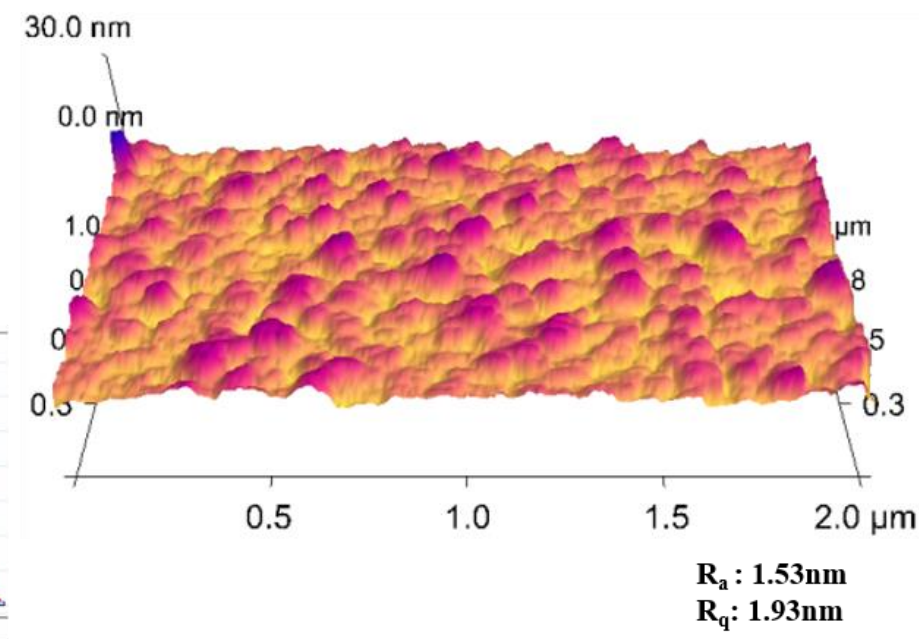

Figure 2. Analysis of the SiN film properties on a 300mm wafer. (a) Ellipsometric measurement of SiN film thickness uniformity (128 points, 1 wafer). (b) AFM picture of $\mathrm{SiN}$ film top surface $\left(2 * 1 \mu \mathrm{m}\right.$ window, wafer center). $\mathrm{R}_{\mathrm{a}}$ : arithmetic average of surface height deviation. $\mathrm{R}_{\mathrm{q}}$ : root mean square average of height deviation.

\section{DEVICE PERFORMANCE}

In order to assess the SiN platform stability, statistical measurements have been performed on passive building blocks with an automatic optical prober at CEA-LETI. The test structure is presented in Figure 3(a). The photonic circuit starts with Silicon Grating Couplers (GC) for efficient chip-fiber coupling. The optical signal is then transferred in the SiN layer thanks to adiabatic transition tapers as described in section 3.2 of this paper. A Y-junction splits the signal on two outputs, one that contains the tested device (OUTD), and another acting as a reference (OUTR) for measurement setup de-embedding. Device characterization are performed by displacing the wafer chuck under a Fiber Array (FA) aligning the fibers with the on-chip grating couplers allowing for fast and reliable characterizations. However, a critical parameter for measurement precision is the initial alignment between the wafer and the fiber array, especially the tilt angle along the vertical $\mathrm{z}$ axis. Figure 3(b) shows a schematic top view of a fiber array on top of Si grating couplers with a tilt angle denoted $\varphi$. As shown in Figure 3(c), this leads to a misalignment in the y direction, denoted $\Delta y_{\text {align }}$, between the fibers and the GC center. In this example, the OUTR fiber is located further to the GC center compared to the OUTD fiber, so $\Delta y_{\text {alignR }}-\Delta y_{\text {alignD }}>0$. From the grating equation ${ }^{21}$, one can see that the GC beam output angle, denoted $\theta$, will raise for shorter wavelength and reduce with higher wavelength. Therefore, the coupling efficiency between the GC and a fiber situated further (resp. closer) to the grating center (as OUTR in this example) will be higher at shorter (resp. higher) wavelength and lower at higher (resp. shorter) wavelength.

(a)

$1500 \mu \mathrm{m}$

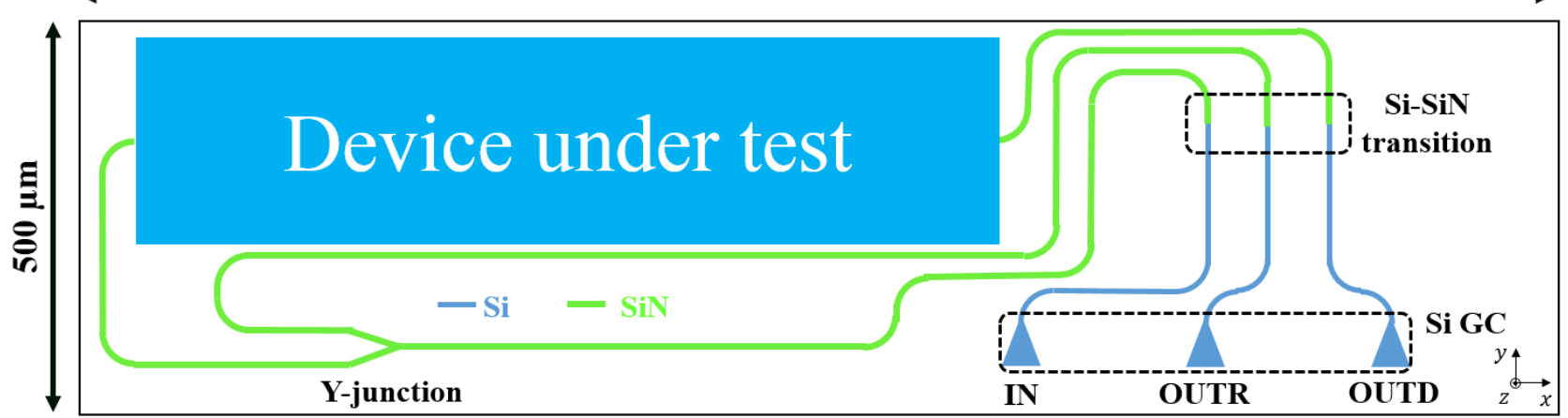



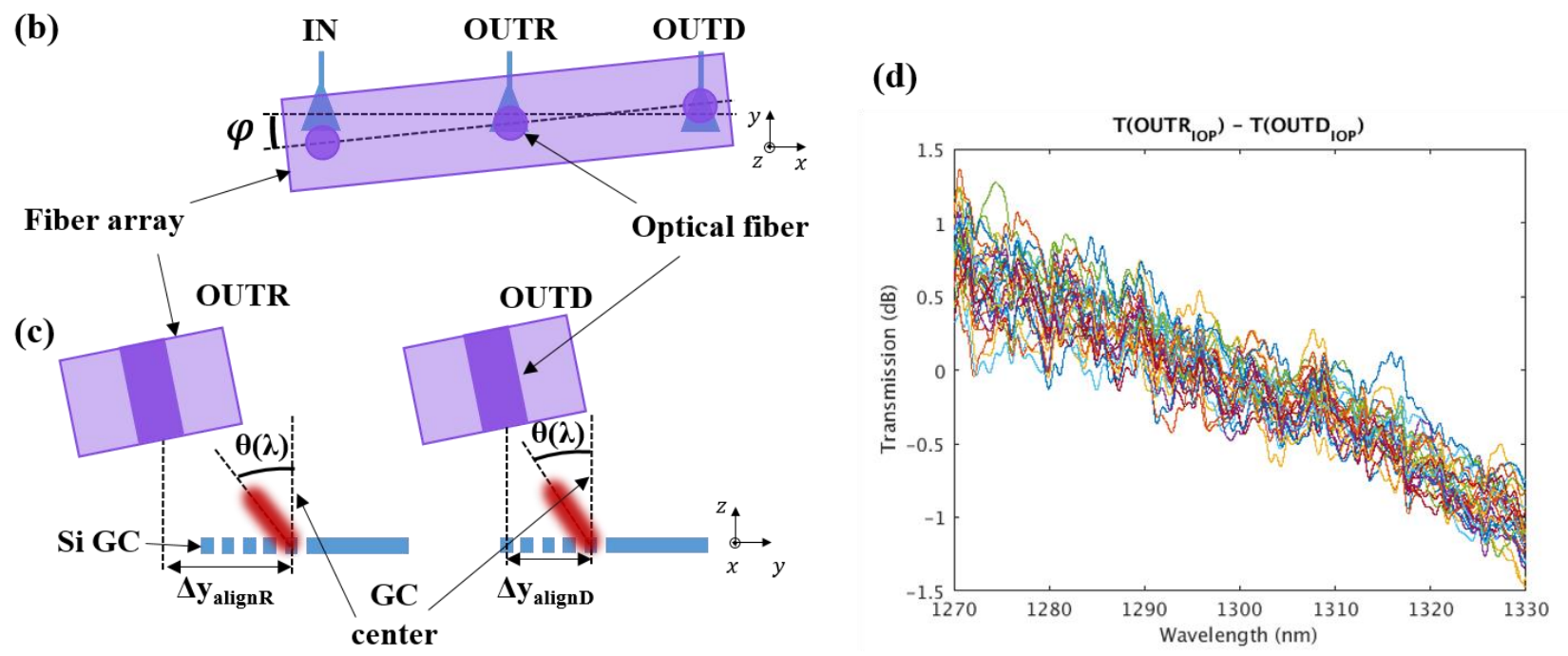

Figure 3. Test structure for statistical measurements. (a) Schematic top view of the testing structure. (b) Schematic top view of the Fiber Array (FA) positioned on top of Si Grating Couplers (GC) revealing tilt angle, $\varphi$, between GC and FA. (c) Schematic side view of fiber array on top of $\mathrm{Si}$ GC revealing the distance from optical fiber to GC center, $\Delta y_{\text {align, for OUTR }}$ $\&$ OUTD. $\theta(\lambda)$ denotes the angle of GC output beam with wavelength. (d) Experimental spectrum of T(OUTRIOP) T(OUTDIOP) for the test structure with Identical Optical Path (IOP) on OUTR \& OUTD (30 die, 1 wafer).

Figure 3(d) shows the experimental response of test structures with identical arms, i.e. OUTR and OUTD have an Identical Optical Path (IOP). The plotted spectrum is the difference between the transmission of OUTR IOP $_{\text {and }}$ OUTD $_{\text {IOP }}$ for 30 die of a single wafer. As one can see, the wavelength-sensitive coupling efficiency is observed on all the measured dies. Therefore, transmission of a Device Under Test (DUT) is obtained with:

$$
\mathrm{T}(\mathrm{DUT})=\left(\mathrm{OUTR}_{\mathrm{DUT}}-\mathrm{OUTD}_{\mathrm{DUT}}\right)-\left(\mathrm{OUTR}_{\mathrm{IOP}}-\mathrm{OUTD}_{\mathrm{IOP}}\right)
$$

The first term of equation (1) stands for test structure de-embedding (grating coupler, Si-SiN and propagation losses normalization) and the second term for wavelength-sensitive coupling efficiency compensation.

\section{1 $\mathrm{SiN}$ waveguide and bend}

Figure 4(a) shows the extracted propagation losses of $700 * 600 \mathrm{~nm}$ single mode SiN strip waveguide in TE polarization for 30 Die of a single wafer.

(a)

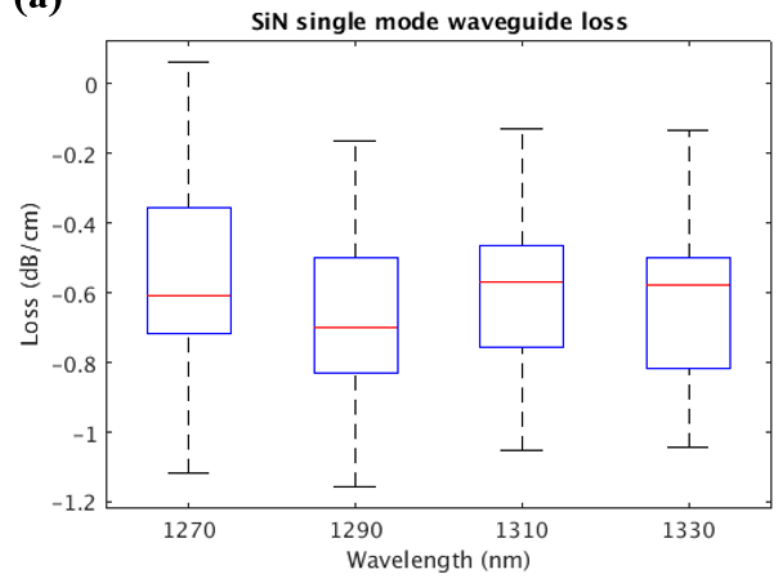

(b)

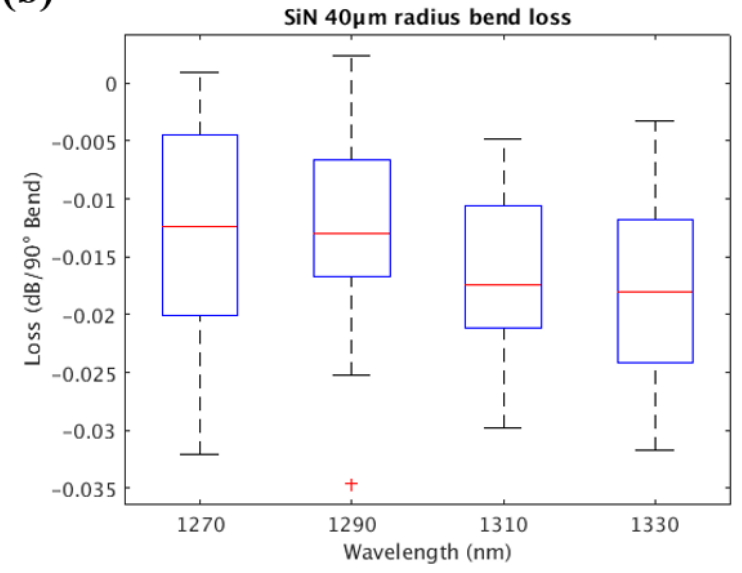

Figure 4. Statistical measurements of SiN waveguide and bend. (a) Single mode TE-polarization SiN waveguide propagation loss (30 die, 1 wafer). (b) SiN 40 $\mu$ m radius TE-polarization bending loss (30 die, 1 wafer). 
Average losses below $0.7 \mathrm{~dB} / \mathrm{cm}$ have been demonstrated for the four wavelength channels of O-band CWDM. Figure 4(b) shows the measured insertion losses of $40 \mu \mathrm{m}$ radius $\mathrm{SiN} 90^{\circ}$ bend waveguide in TE polarization for $30 \mathrm{Die}$ of a single wafer. Average losses below $0.02 \mathrm{~dB} / 90^{\circ}$ have been obtained for the four wavelength channels of O-band CWDM.

\section{2 $\mathrm{Si}-\mathrm{SiN}$ adiabatic transition}

Optical power transfer between the two photonic layers is ensured by adiabatic linear tapers as depicted schematically on Figure 5(a). Progressive reduction of the Si and SiN waveguides width allows for maximum efficiency by de-confining the optical modes as well as matching the respective effective indexes. An Eigen Mode Expansion (EME) solver [REF] have been used for device simulation. As shown on Figure 5(b), a good trade-off between device length and transmission efficiency can be found for a taper length of $90 \mu \mathrm{m}$

(a)

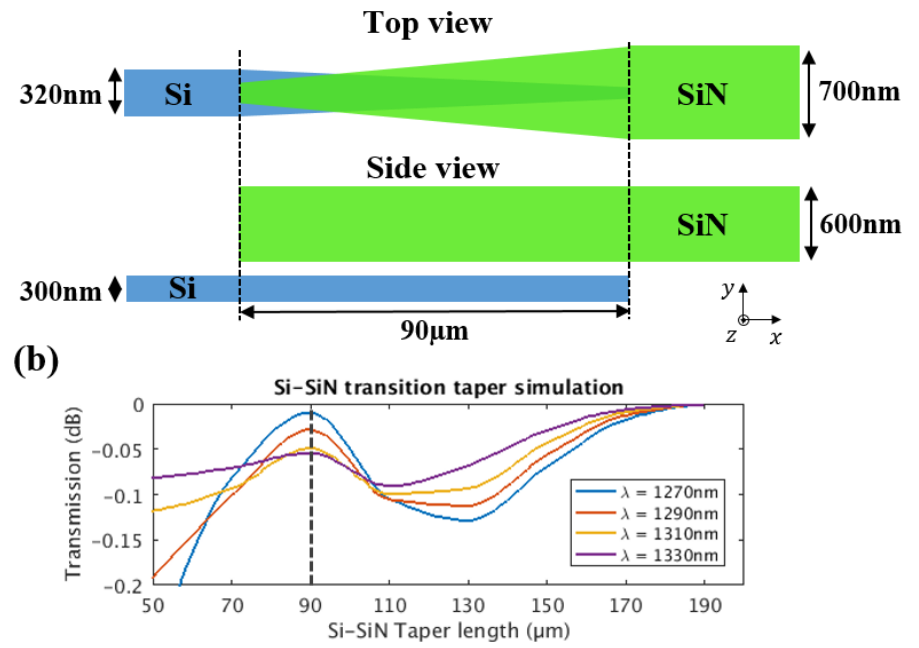

(c)

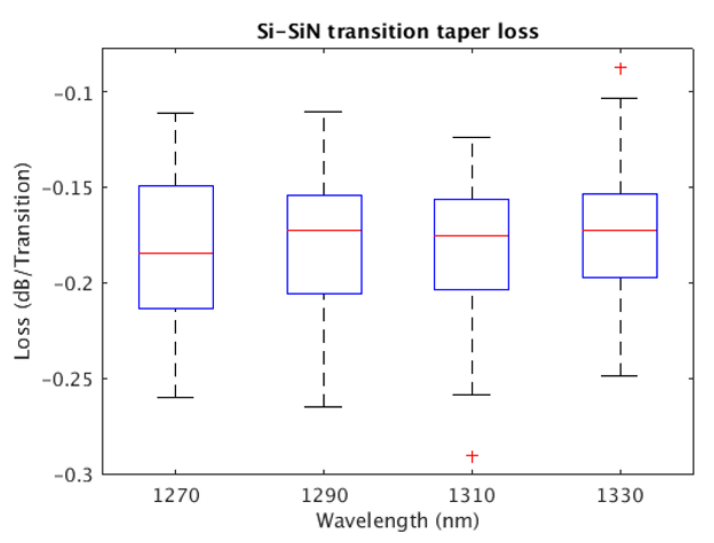

Figure 5. Development of an adiabatic Si-SiN transition taper. (a) Schematic view of the transition taper. (b) Numerical simulation (EME solver ${ }^{22}$ ) of the taper length impact on transmission performance. (c) Statistical measurement of $\mathrm{Si}-\mathrm{SiN}$ $90 \mu \mathrm{m}$ transition loss (30 die, 1 wafer).

Figure 5(c) shows the measured transition loss on 30 die of a single wafer. Average transition losses below 0.17 $\mathrm{dB}$ was obtained for the four wavelength channels of O-band CWDM. Misalignment between the Si and SiN structures have been measured in line for all die present on the wafer revealing a three sigma of $41 \mathrm{~nm}$ (resp. $39 \mathrm{~nm}$ ) in the $\mathrm{x}$ (resp. y) direction as defined in Figure 5(a). This alignment deviation partially explains the transition loss dispersion.

\section{3 $\mathrm{SiN}$ wavelength stabilized directional coupler}

Power splitters are essential building blocks of silicon photonic circuits. Directional Coupler (DC) is the most common one, demonstrating an easily tunable splitting ratio and a compact footprint. However, it present a very large sensitivity to the operating wavelength making its used complicated for large wavelength range applications such as CWDM. As detailed in $^{23}$, wavelength-stabilized directional couplers (WSDC) can be obtained by inserting a phasecontrol section between two standard DC. A schematic top view of the WSDC is shown on the inset of Figure 6(b). By a proper tuning of the phase control section length $\mathrm{L}_{\mathrm{pc}}$ and standard DC length $\mathrm{L}_{\mathrm{c}}$, one can obtain stabilization of the overall device splitting ratio. As proposed $\mathrm{in}^{23}$, device modeling have been performed through a semi analytical model based on Transfer Matrix Modeling (TMM) and further optimized by 3-D FDTD simulation from ${ }^{22}$.

Measurements of WSDC splitting ratio have been performed through the extinction ratio of an asymmetric MZI with the WSDC as the output coupler ${ }^{23}$. Figure 6(a) shows the spectral behavior of a 10/90 WSDC measured in TE polarization. The red and blue curves are the two outputs of the MZI. As shown, the splitting ratio of the WSDC is extracted at each resonance of the MZI (denoted by the vertical dashed lines). An average splitting ratio of $90.3 \%$ is obtained with a maximum deviation of less than $3.5 \%$ on the whole O-band. Figure 6(b) shows the measured splitting ratio of a 50/50 WSDC in TE polarization. As shown, an average splitting ratio of $52.3 \%$ is obtained with a maximum deviation of less than $4.8 \%$ on the whole O-band. The deviation of the mean extinction ratio is attributed to the residual 
level of TM polarization that is not properly filtered by the measurement setup which limits the extinction ratio of the MZI i.e. the measured WSDC splitting ratio (since perfect 50/50 splitting ratio requires infinite MZI extinction ratio).

(a)

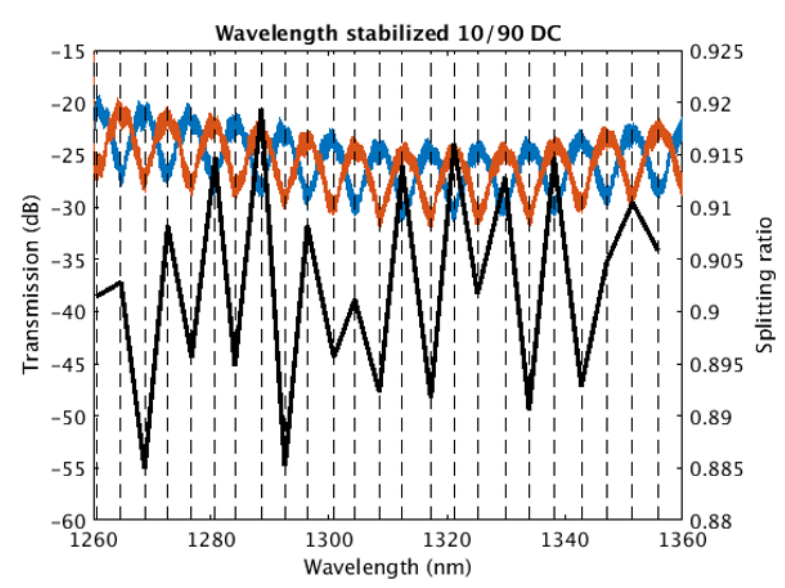

(b)

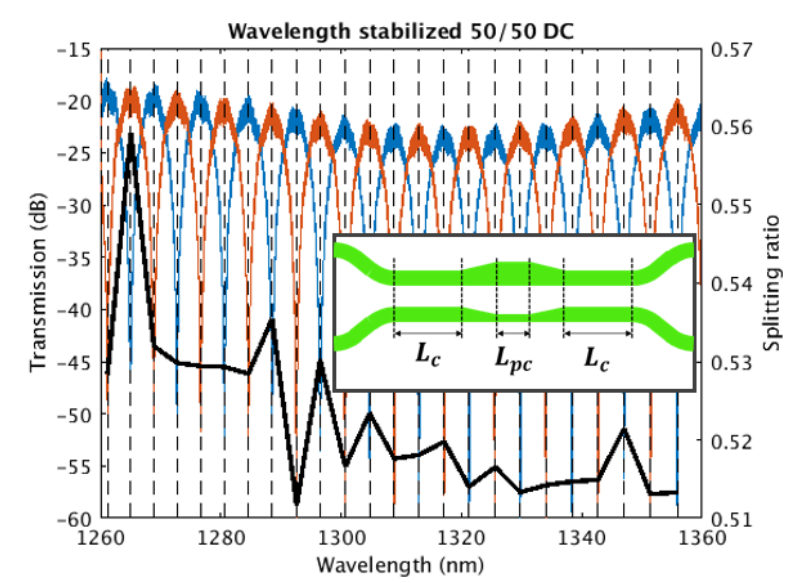

Figure 6. Development of SiN Wavelength-Stabilized Directional Couplers (WSDC). (a) Experimental measurement of a 10/90 WSDC spectral behavior in TE polarization. The red and blue curves are the transmission of the unbalanced MZI, the black curve is the extracted WSDC splitting ratio. (b) Experimental measurement of a 50/50 WSDC spectral behavior in TE polarization. The small inset is a schematic top view of the WSDC with $\mathrm{L}_{\mathrm{c}}$ the coupling length and LPC the phase control section length.

\section{CONCLUSION}

In conclusion, we have demonstrated co-integration of a Silicon Nitride photonics layer within the ST 300mm R\&D platform DAPHNE. The SiN film have been characterized through ellipsometry and AFM showing a good thickness uniformity and a small top surface roughness. Statistical measurements have been performed on key building blocks such as $\mathrm{SiN}$ strip waveguides showing losses below $0.7 \mathrm{~dB} / \mathrm{cm}$ and $40 \mu \mathrm{m}$ radius bends with losses below $0.02 \mathrm{~dB} / 90^{\circ}$. A compact $\mathrm{Si}-\mathrm{SiN}$ transition taper have been developed and statistically measured showing insertion losses below $0.17 \mathrm{~dB} /$ transition on the whole O-band. Targeting CWDM applications, WSDC in SiN have been developed showing stable splitting ratio all other the O-band. Advanced passive devices are currently under development such as MUX/DEMUX based on AWG and Echelle Gratings, polarization management devices, polarization insensitive power splitters and high $\mathrm{Q}$ ring resonators.

\section{ACKNOWLEDGEMENTS}

This work was partially funded by the European Commission through the H2020-ICT-27-2017 COSMICC project under the grant agreement $n^{\circ} 688516$ and by European Research Council (ERC) under the European Union's Horizon 2020 Research and Innovation Program (ERC POPSTAR, grant agreement $n^{\circ} 647342$ ). We also acknowledge financial support from the French Industry Ministry Nano2017 program.

\section{REFERENCES}

[1] Perez-Galacho, D., Baudot, C., Hirtzlin, T., Messaoudène, S., Vulliet, N., Crozat, P., Boeuf, P., Vivien, L., Marris-Morini, D., "Low voltage 25Gbps silicon Mach-Zehnder modulator in the O-band", Optics Express 25(10), 11217-11222 (2017).

[2] Virot, L., Crozat, P., Fédéli, J-M., Hartmann, J-M., Marris-Morini, D., Cassan, E., Bœuf, F., Vivien, L., "Germanium avalanche receiver for low power interconnects", Nature Communications 5, (2014). 
[3] Barwicz, T., Janta-Polczynski, A., Khater, M., Thibodeau, Y., Leidy, R., Maling, J., Martel, S., Engelmann, S., S. Orcutt, J., Fortier, P., M. J. Green, W., “An O-band Metamaterial Converter Interfacing Standard Optical Fibers to Silicon Nanophotonic Waveguides", Optical Fiber Communications Conference and Exhibition (OFC), (2015).

[4] Benedikovic, D., Alonso-Ramos, C., Pérez-Galacho, D., Guerber, S., Vakarin, V., Marcaud, G., Le Roux, X., Cassan, E., Marris-Morini, D., Cheben, P., Boeuf, F., Baudot, C., Vivien, L., "L-shaped fiber-chip grating couplers with high directionality and low reflectivity fabricated with deep-UV lithography", Optics Letters 42(17), 3439-3442 (2017).

[5] CISCO, "Global Cloud Index 2016-2021: Forecast and Methodology", 1 February 2018, <https://www.cisco.com/c/en/us/solutions/collateral/service-provider/global-cloud-index-gci/white-paper-c11738085.html> (1 February 2018).

[6] Bœuf, F., Crémer, S., Vulliet, N., Pinguet, T., Mekis, A., Masini, G., Verslegers, L., Sun, P., Ayazi, A., Hon, N., Sahni, S., Chi, Y., Orlando, B., Ristoiu, D., Farcy, A., Leverd, F., Broussous, L., Pelissier-Tanon, D., Richard, C., Pinzelli, L., Beneyton, R., Gourhant, O., Gourvest, E., Le-Friec, Y., Monnier, D., Brun, P., Guillermet, M., Benoit, D., Haxaire, K., Manouvrier, J.R., Jan, S., Petiton, H., Carpentier, J.F., Quémerais, T., Durand, C., Gloria, D., Fourel, M., Battegay, F., Sanchez, Y., Batail, E., Baron, F., Delpech, P., Salager, L., De Dobbelaere, P., Sautreuil, B., "A multi-wavelength 3D-compatible silicon photonics platform on 300mm SOI wafers for 25Gb/s applications", IEEE International Electron Devices Meeting (IEDM), 1331-1334 (2013).

[7] R. Doerr, C., Chen, L., Vermeulen, D., Nielsen, T., Azemati, S., Stulz, S., McBrien, G., Xu, X-M., Mikkelsen, B., Givehchi, M., Rasmussen, C., Park, S-Y., "Single-Chip Silicon Photonics 100-Gb/s Coherent Transceiver", Optical Fiber Communication Conference: Postdeadline Papers, (2014).

[8] Guerber, S., Alonso-Ramos, C., Perez-Galacho, D., Le Roux, X., Vulliet, N., Crémer, S., Marris-Morini, D., Boeuf, F., Vivien, L., Baudot, C., "Design and integration of an O-band silicon nitride AWG for CWDM applications", Proc. of IEEE 14-th International Conference on Group IV Photonics (GFP), 133-134 (2017).

[9] Pathak, S., Van Thourhout, D., Bogaerts, W., "Design trade-offs for silicon-on-insulator-based AWGs for (de)multiplexer applications", Optics Letters 38(16), 2961-2964 (2013).

[10] Hassan, K., Sciancalepore, C., Harduin, J., Ferrotti, T., Menezo, S., Ben Bakir, B., "Toward athermal siliconon-insulator (de)multiplexers in the O-band", Optics Letters 40(11), 2641-2644 (2015).

[11] Wu, H., Tan, Y., Dai, D., "Ultra-broadband high-performance polarizing beam splitter on silicon", Optics Express 25(6), 6069-6075 (2017).

[12] Chen, D., Xiao, X., Wang, L., Liu, W., Yang, Q., Yu, S., "Highly efficient silicon optical polarization rotators based on mode order conversions", Optics Letters 41(5), 1070-1073 (2016).

[13] Soldano, L., Kubicky, J., Ton, D., Wendland, J., Allen, M., Grant, A., Pezeshki, B., "Multi-wavelength 100Gb/s Silicon Photonics Based Transceiver with Silica mux/demux and MEMS-coupled InP Lasers", Proc. Optical Fiber Communication Conference, paper Th3B.1 (2017).

[14]Zhang, Z., Yako, M., Ju, K., Kawai, N., Wada, K., “A Silicon Nitride Platform by Physical Vapor Deposition for Dense Wavelength Division Multiplexing on Chip", Proc. of IEEE 11-th International Conference on Group IV Photonics, 193-194 (2014).

[15] Rahim, A., Ryckeboer, E., Z. Subramanian, A., Clemmen, S., Kuyken, B., Dhakal,Ali Raza, A., Hermans, A., Muneeb, M., Dhoore, S., Li, Y., Dave, U., Bienstman, P., Le Thomas, N., Roelkens, G., Van Thourhout, D., Helin, P., Severi, S., Rottenberg, X., Baets R., "Expanding the Silicon Photonics Portfolio with Silicon Nitride Photonic Integrated Circuits", IEEE Journal of Lightwave Technology 35(4), 639-649 (2017).

[16] Geidel, S., Llopis, S-P., Rodrigo, M., Diego-Castilla, G., Sousa, A., Nestler, J., Otto, T., Gessner T., Parro, V., "Integration of an Optical Ring Resonator Biosensor into a Self-Contained Microfluidic Cartridge with Active, Single-Shot Micropumps", Micromachines 7(9), 153 (2016).

[17] Rönn, J., Zhang, W., Autere, A., Le Roux, X., Durán-Valdeiglesias, E., Pakarinen, L., Baudot, C., Guerber, S., Boeuf, F., Alonso-Ramos, C., Säynätjoki, .A, Vivien, L., Cassan, E., Sun, Z., "On-chip optical amplification at C-band in silicon nitride slot waveguides", SPIE, Accepted (2018).

[18] Marin-Palomo, P., N. Kemal, J., Karpov, M., Kordts, A., Pfeifle, J., H. P. Pfeiffer, M., Trocha, P., Wolf, S., Brasch, V., H. Anderson, M., Rosenberger, R., Vijayan, K., Freude, W., J. Kippenberg T., Koos, C., "Microresonator-based solitons for massively parallel coherent optical communications", Nature 546, 274-279 (2017).

[19] Baudot, C., Fincato, A., Fowler, D., Perez-Galacho, D., Souhaité, A., Messaoudène, S., Blanc, R., Richard, C., Planchot, J., De-Buttet, C., Orlando, B., Gays, F., Mezzomo, C., Bernard, E., Marris-Morini, D., Vivien, L., 
Kopp, C., Bœuf, F., "DAPHNE silicon photonics technological platform for research and development on WDM applications", Proc. SPIE 9891, (2016).

[20] Domínguez Bucio, T., Z Khokhar, A., Lacava, C., Stankovic, S., Z Mashanovich, G., Petropoulos P., Y Gardes, F., "Material and optical properties of low temperature NH3 free PECVD SiN layers for photonic applications", Journal of Physics D: Applied Physics 50(2), (2016).

[21] T. Reed, G. and P. Knights, A., [Silicon Photonics: An Introduction], 2004 John Wiley \& Sons, Ltd, 80 (2005).

[22] Lumerical Solutions Inc., 2018, <https://www.lumerical.com> (2018).

[23]Lu, Z., Yun, H., Wang, Y., Chen, Z., Zhang, F., A. F. Jaeger, N., Chrostowski, L., "Broadband silicon photonic directional coupler using asymmetric-waveguide based phase control”, Optics Express 23, 3795-3808 (2015). 